\title{
Water Monitoring Robot with Double Layer Structure Design
}

\author{
Shuai Zhao ${ }^{1}$, Boyun Liu ${ }^{2}$, Yuanju Jian ${ }^{1}$, Yi Yuan ${ }^{2}$ and Shangxuan Xiao ${ }^{1}$ \\ ${ }^{1}$ College of Electrical Engineering, Naval University of Engineering, Wuhan, 430033 \\ ${ }^{2}$ College of Power Engineering, Naval University of Engineering, Wuhan, 430033
}

\begin{abstract}
A surface patrol robot with separate upper and lower structure and equipped with a water meter and lifesaving equipment throwing device is proposed. The robot can patrol scheduled water area, monitor water quality in real-time, rescue drowning persons and perform other tasks. The robot is modular designed and mainly include six main modules i.e. image transmission, satellite navigation, water quality monitoring, rescue, power supply and control system. The robot has advantages of good seakeeping and extensive adapt so can be widely applied in water management and other fields.
\end{abstract}

Keywords-river patrol; drowning rescue; water sample detection; intelligent navigation

\section{INTRODUCTION}

In recent years, the country vigorously develop the environmental engineering construction, especially in natural waters of governance, Many countries also attach great importance to water environment governance, and design a series of UUV [1] to replace divers for performing exploration and investigation work of waters, such as China "Dragon", the "Tuna" [2], For the lifesaving device delivery to personnel on the water in the complex water conditions, China underwater robot research expert Yan Kuichenhad made a rescue robot in 2000 for used of surface salvage [3].

Aiming at three problems of governance of natural waters - real-time monitoring channel[4], real-time monitoring of water [5] and make emergency rescue of accidental drowning personnel[6],we developed a robot equipped with water quality measuring instrument and simple life-saving device, Next we will illustrate the function, the mechanical structure and working principle of the robot.

\section{STRUCTURAL DESIGN}

As shown in Fig. 1, the river patrol robot adopts the structure design of separation between the upper and the lower. The shape parameter of the main body and the appendage has excellent stability, and can adapt to the complex rescue environment. The water drop shape of the carrier cabin can reduce the navigation resistance and rapid mobility.
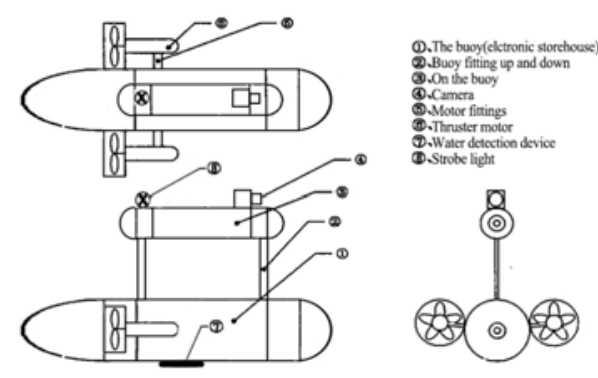

FIGURE I. STRUCTURE SCHEMATIC DIAGRAM

\section{A. Carrier Cabin Design}

The main part of the robot is similar to that of the underwater vehicle, and the shape of the underwater vehicle can be referenced [7]. A preliminary estimate of the amount of water required for a vehicle to be neutral in water:

$$
\mathrm{V}=\frac{M}{\rho}=\frac{50 \mathrm{~kg}}{1 \mathrm{~kg} / \mathrm{L}}=50 \mathrm{~L}
$$

$M$ is the weight of robot, $\rho$ is the liquid density for navigating a robot,

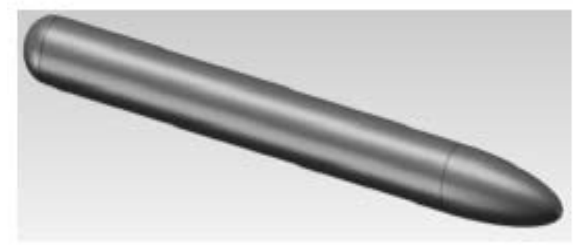

FIGURE II. MAIN BODY

In Fig. 2, the first end of the body is a hemisphere of diameter $0,2 \mathrm{~m}$, tail correction for eccentricity e 3.5 semiellipsoid, the middle part is a cylinder with the diameter of $0,2 \mathrm{~m}$ as shown in Fig. 3, the processing is simple, low cost, high space utilization, has better hydrodynamic performance, The following are the parameters of the main body [8], as shown in Tab 1: 


\begin{tabular}{ll}
\hline \multicolumn{1}{c}{ Parameter } & \multicolumn{1}{c}{ Numerical value } \\
\hline Length & $1,72 \mathrm{~m}$ \\
Weight & $50 \sim 60 \mathrm{~kg}$ \\
Fuselage & Diameter:0,2m Linear revolving body \\
& \\
Propeller & Disk ratio:0,35 Maximum diameter0,15m \\
& Half extension length: $0,12 \mathrm{mAspect}$ ratio: 1,2 \\
Rudder & Sweep angle $15^{\circ}$ Linear: NACA0012 \\
\end{tabular}

\section{B. Propulsion System Design And Design}

The propulsion system is designed to use the ducted propeller, which is provided with a sleeve or catheter outside the open water propeller. The section of the duct is wing shaped. The outer side is straight, and the inner part is curved, When the propeller rotates forward, the duct produces a negative pressure zone in the front of the rotating plane of the propeller, producing a positive pressure zone at the rear, so that a forward thrust can be obtained, The static thrust of the catheter is the maximum when the velocity is zero, when the robot starts moving from the stationary position, the efficiency is the highest, At low speed, ducted propellers can increase efficiency by $20 \%$, so it is particularly suitable for patrol robots.

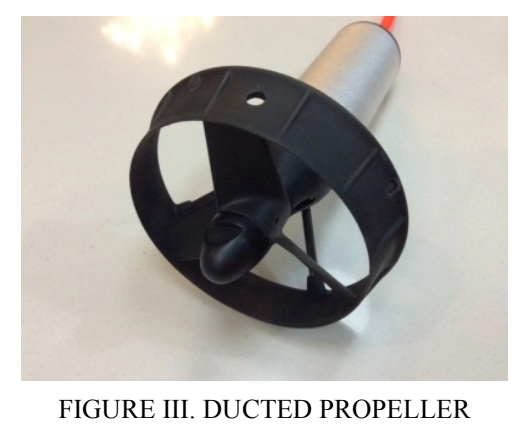

\section{CONTROL SYSTEM DESIGN}

The robot control system has two control modes, automatic navigation mode and remote control mode [9], This system adopts NXP K60 MCU as the main control unit of the robot, using NEO-M8N Pixhawk APM as a navigation controller, FPV700 single board HD camera as the camera sensor to collect image information around the robot, The image information receiving image module and transmit information through image transmission module to the host computer then display it on the PC screen.

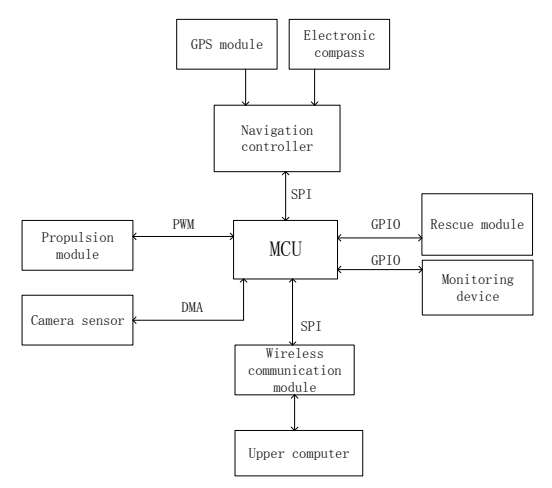

FIGURE IV. CONTROL DIAGRAM

When the robot is patrolling, the automatic navigation mode is opened and the robot navigates on the surface according to the planned route of the operator, A monitor screen is installed on the upper computer of the robot to display the image information collected by the camera sensor at all times, The operators by opening remote mode make judgment through observing information is displayed on the screen to decide whether to disable the automatic navigation mode, through the control of the equipment, thus the robot could complete the special tasks such as emergency rescue personnel on the water. 


\section{FUNCTION REALIZATION}

\section{A. River Dynamic Monitoring}

The robot has a vision sensor at the front of the robot's water structure, The sensor collects the image around the robot and sends the signal to the MCU, After the signal processing, the MCU transfers the signal to the host computer in real time, In this way, the image captured by the camera is fed back to the operator so that the operator can control the condition around the river at any time.

\section{B. Intelligent Navigation and Path Planning}

The geographic coordinates of several points are measured in the water area where the robot needs to patrol, such as Fig,7, and the coordinates are input into the upper computer in order to store and plan the planned navigation line of the robot, The robot has the connotation of the navigation controller GPS positioning device and compass to the host computer to send the robot's location and robot course, while computer calculates the coordinates where the robot and target coordinate azimuth error and trajectory direction [10], By controlling the rudder angle of robot and the power of two thrusters, the heading of the robot is adjusted to the course of target, It reduces the workload of the operator.

The robot collects the image information through the visual sensor and transmits it to the MCU, The MCU sends the image information to the host computer, By observing the monitoring screen on the upper computer, the operator can understand the river condition, Once the special situation occurs, the operator can relieve the robot's automatic navigation mode and change it into remote control mode, Through the simple key operation of the host computer, the robot is controlled by human, so that the robot can complete the special task.

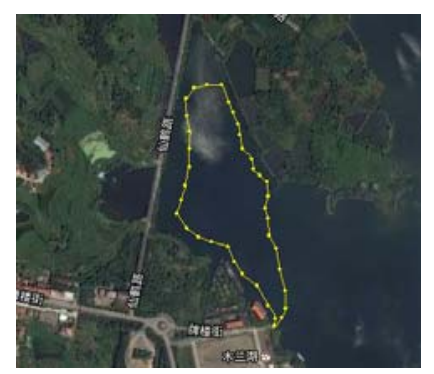

FIGURE V. PLANNED ROUTE BY GPS

\section{Real Time Monitoring of Water Quality}

The underwater part of the robot is equipped with a self-developed water sampling device, which is placed in the electronic cabin of the robot, with three probes placed at the bottom of the underwater part of the robot, The robot can carry out the real-time detection indexes for waters water $\mathrm{pH}$, conductivity, solubility of organic compounds and temperature during the voyage, and real-time display in the host computer. Once the water body appears strange, the PC will sound the alarm and record of robot location and water's data.

\section{Throwing of Lifesaving Equipment}

The pneumatic throwing device is installed at the front end of the water part of the robot, The opening is closed when the patrol is in progress, and the opening is opened when the emergency state is in place, When the drowning person is in need of assistance, it will be cast to the transmitter in front of goal, and the rotating handle will hit pierced cylinders, while the gas chamber will be filled with gas quickly, to throw the life buoy to the rescue target, when the inflatable life buoy is exposed to water, the automatic inflating device will be inflated in 5 seconds, and the gas cylinders filled with gas chamber, with the gas pressure the gas chamber will be separated from the shell and automatically inflated, with automatic inflatable molding buoy posture floating on the water, acting as the role of lifesaving [11].

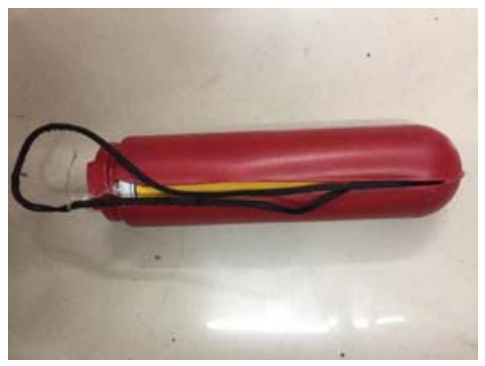

FIGURE VI. THE AUTOMATIC INFLATABLE BUOY

\section{CONCLUSION}

(1) Due to the robot uses a nearly fully submerged carrier structure, water surface wind and ocean current have a tiny impact on the robot. The design of separate upper and lower structure for the robot improves its metacenter; the weight's concentration in the central electronic cabin of underwater robot makes the overall structure a "Tumbler" with a center of mass in underwater central electronic cabin which has strong sailing stability,

(2) The robot can navigate and patrol autonomously in regulated waters, and the pattern reduces the technical requirement of the operator. The implementation of dynamic monitoring in the waters can not only be used in static monitoring of the monitoring points but also inspect in a full range flexibly, take pictures of evidence and monitor water sample in real time to the monitoring points, which improves the supervision and reduces the economic cost of environmental monitoring. The added rescue module greatly improves the safety of water security personnel. The flexible and maneuverable rescue mode can reduce the probability of the occurrence of drowning in the water,

(3) In the past, taking water samples requires sampling personnel going to the predetermined waters by boat which cost large human and material resources and the boat itself has pollution to the water. The robot uses aluminum alloy materials which are light and economic; the power system uses lithium batteries to provide energy, which reduces the consumption of human and material resources and has tiny pollution to the environment. 


\section{REFERENCE}

[1] Defense Science Board, Next-Generation Unmanned Undersea Systems[R], U,S,: Office of the Secretary of Denfense, 2016.

[2] Qian Dong, Tang Xian-ping ,Zhao Jiang ,Overview of Technology Development and System Design of UUVs[J], TORPEDO TECHNOLOGY: 2014(12): 401-419.

[3] Yan Kui-chen, Yuan Xue-qing, Qin Bao-cheng. A SURFACE RESCUE VEHICLE[J]. ROBOT: 2001(11): 23(6): 493-497.

[4] Gao Dui-dui, Shao Ming. Application of monitoring technology in river regulation and river management[J]. Zhejiang Hydrotechnics: 2002(03): 41-42.

[5] Li Zheng-hong ,Guo Xiu-hong,Wang Shan,Technical Features of Potable Water Quality Analyzer and Quality Assured[J], JOURNAL OF CHINA HYDROLOGY: 2006(12):26(6): 57-59.

[6] Zhang Jia-ming. Discussion on rescue of falling water accident in Chinese $[\mathrm{J}]$. Fire protectiontechnology and product information in Chinese2012(11):20

[7] Jiang Xin-song, FENG Xi-sheng, WANG Ti-tang, UNMANNED UNDERWATER VEHICLES[M],Shenyang science and Technology Press:2000:53

[8] Han Xiao-dong, Wang Jian-ru, Meng Xiu-qing, Underwater navigation body numerical simulation of influence on the resistance[J], Research And Design2011: 38(9)15-22.

[9] Liao Yu-lei, Pang Yong-jie, ZHUANG Jia-yuan, Study of the Embedded Basic Motion Control System for an Unmanned Surface Vehicle[J], Computer Science 2010(10): 37(9)214-217.

[10] Zhan Lei, He Ren-qing Xie Yang, Long Yan, Intelligent navigation system based on four-rotor micro UAV[J], ELECTRONIC MEASUREMENT TECHNOLOGY: 2001(6): 34(6): 1-4.

[11] Ou Yang-lei, Gu Lei, Development and application of a new type of long distance lifesaving throw off device in Chinese[J],Fire protection technology and product in formationin Chinese 2012: 12

[12] Qian Dong, Meng Qing-guo, Xue Meng, et al, Requirements for Task and Capability of the US Navy Unmanned Underwater Vehicles[J], Torpedo Technology, 2005, 13(4): 7-12 